\title{
Airway Hyperreactivity Is Delayed after Mild Neonatal Hyperoxic Exposure
}

\author{
Harris Onugha Peter M. MacFarlane Catherine A. Mayer Akua Abrah \\ Anjum Jafri Richard J. Martin \\ Division of Neonatology, Rainbow Babies and Children's Hospital, and Department of Pediatrics, \\ Case Western Reserve University, Cleveland, Ohio, USA
}

\section{Key Words}

Hyperoxia · Wheezing · Preterm infants · Airway

hyperreactivity $\cdot$ Neonatal mouse

\begin{abstract}
Background: Wheezing disorders are prominent in former preterm infants beyond the neonatal period. Objectives: We used a neonatal mouse model to investigate the time course of airway hyperreactivity in response to mild ( $40 \%$ oxygen) or severe (70\% oxygen) neonatal hyperoxia. Methods: After hyperoxic exposure during the first week of postnatal life, we measured changes in airway reactivity using the in vitro living lung slice preparation at the end of exposure [postnatal day 8 (P8)] and 2 weeks later (P21). This was accompanied by measures of smooth muscle actin, myosin light chain (MLC) and alveolar morphology. Results: Neither mild nor severe hyperoxia exposure affected airway reactivity to methacholine at P8 compared to normoxic controls. In contrast, airway reactivity was enhanced at P21 in mice exposed to mild (but not severe) hyperoxia, 2 weeks after exposure ended. This was associated with increased airway a-smooth muscle actin expression at P21 after $40 \%$ oxygen exposure without a significant increase in MLC. Alveolar morphology via radial alveolar counts was comparably diminished by both 40 and $70 \%$ oxygen at both P8 and P21. Conclusions: These data
\end{abstract}

demonstrate that early mild hyperoxia exposure causes a delayed augmentation of airway reactivity, suggesting a longterm alteration in the trajectory of airway smooth muscle development and consistent with resultant symptomatology.

(c) 2015 S. Karger AG, Basel

\section{Introduction}

Wheezing disorders and asthma remain a major source of longer-term morbidity in former preterm infants [1,2]. While this is most severe in infants with a history of bronchopulmonary dysplasia (BPD), even late preterm infants appear at risk, many of whom may only have exposure to modest supplemental oxygen [3-5]. Interestingly, the increased airway reactivity exhibited by former preterm infants usually manifests beyond the neonatal period as exemplified by the limited benefit for bronchodilator therapy in the neonatal intensive care unit [6]. Therefore, there is a need for animal models to characterize the developmental trajectory of airway function after neonatal lung injury.

Neonatal rodents have served as models to study respiratory mechanics after neonatal hyperoxia exposure and lung mechanics or airway hyperreactivity have been documented in vivo from 14 and 21 days of life [7-12]. Com-

\section{KARGER 125}

(C) 2015 S. Karger AG, Basel

$1661-7800 / 15 / 1081-0065 \$ 39.50 / 0$

E-Mail karger@karger.com

www.karger.com/neo
Richard J. Martin, MD

Department of Pediatrics

Case Western Reserve University, Rainbow Babies and Children's Hospital

11100 Euclid Avenue, Cleveland, OH 44106-6010 (USA)

E-Mail rxm6@case.edu 
parative data on lung development indicate that alveolar formation in rodents is largely complete by day 14 , which approximates the lung of a 2 -year-old infant $[13,14]$. Unfortunately, the small size of neonatal mice precludes reliable in vivo measurement of airway reactivity prior to approximately day 14 . However, the living lung slice model provides a novel technique to quantify changes in airway caliber in response to a bronchoconstrictor challenge under in vitro conditions [15]. This method allows airway contractility to be assessed with bronchoalveolar attachments intact as would occur in vivo, and we have adapted this technique to study lung slices in 1-week-old mice.

In this study we exposed mouse pups to mild (40\% oxygen) or severe (70\% oxygen) hyperoxia for the first 7 postnatal days and used living lung slices at postnatal $(\mathrm{P})$ days 8 and 21 to test the following two hypotheses: (1) onset of airway hyperreactivity is delayed after neonatal hyperoxic exposure, and (2) mild hyperoxic exposure causes a greater increase in airway reactivity than severe hyperoxia as suggested by our prior in vivo studies employing lung mechanics. We also sought to correlate our physiologic data with changes in airway smooth muscle and lung morphology.

\section{Methods}

All procedures were carried out in accordance with the National Institutes of Health guidelines for laboratory animals and approved by the Animal Care and Use Committee at Case Western Reserve University. Time-pregnant mice (C57BL/6J) gave birth in our animal facility and together with the dam were assigned to receive 40 or $70 \%$ oxygen or room air for 7 consecutive days (control). Measurements of airway responses to methacholine using living lung slice preparation [15] were performed on 8 or 9 airways of comparable size per pup on either the day after exposure ended (P8) or at P21 (2-week recovery in room air). We studied only male mice to avoid an effect of sex on airway hyperresponsiveness [16].

For immunohistochemistry and morphology, the trachea was cannulated and the lungs were inflation fixed $\left(25 \mathrm{~cm} \mathrm{H}_{2} \mathrm{O}\right)$ for 10 min with $10 \%$ neutral-buffered formalin as we have previously described [11]. The left lung was removed and postfixed for 2 days at $4^{\circ} \mathrm{C}$ and embedded in paraffin, and $5-\mu \mathrm{m}$ paraffin sections were processed. In those studies we analyzed 2-3 airways of comparable size $(100-320 \mu \mathrm{m}$ in diameter) in each pup. For all experiments, group sizes comprised 5-7 pups.

\section{Hyperoxia Exposure}

Hyperoxic exposure consisted of the dam and her litter being placed inside a plexiglass chamber $(30 \mathrm{~L} \times 50 \mathrm{~W} \times 28 \mathrm{H} \mathrm{cm})$, through which gas flowed $(3 \mathrm{l} / \mathrm{min})$ at the desired concentration (room air, 40 or $70 \%$ oxygen). Oxygen concentrations were monitored and achieved using manual flow controllers as we have previously described [11]. Mice were exposed $24 \mathrm{~h} /$ day for 7 consecutive days starting the day after birth (P1). Dams were cycled be- tween litters exposed to room air and hyperoxia every $24 \mathrm{~h}$ to minimize acute oxygen toxicity and to ensure that the normoxic and hyperoxic dams received the same exposure. At the end of the seventh day of exposure, the pups were removed from the chamber and then raised for a further 14 days in normoxia. Cages, water, and food were replaced every 3 days. Normoxic mice received room air for the same time period.

Hyperoxic exposure resulted in a decrease in body weight at P8 after $70 \%$ oxygen exposure in the pups who underwent physiologic study (room air: $5.05 \pm 0.25$ g; $40 \%$ oxygen: $4.56 \pm 0.19$ g; $70 \%$ oxygen: $3.91 \pm 0.3 \mathrm{~g} ; \mathrm{p}<0.02$ ). At P21, body weights no longer differed [room air: $9.92 \pm 1.05$ g; $40 \%$ oxygen: $11.02 \pm 0.36$ g; $70 \%$ oxygen: $8.77 \pm 0.67$ g; n.s.].

\section{Measurement of Airway Reactivity and in vitro Living Lung} Slice Preparation

At P8 and P21, mice were sacrificed via anesthetic overdose, intraperitoneal injection of a ketamine $(500 \mathrm{mg} / \mathrm{kg})$-xylazine (100 $\mathrm{mg} / \mathrm{kg}$ ) mix, to prepare the lungs for in vitro measurements of airway reactivity to methacholine. The trachea was cannulated and agarose gently injected to inflate the lungs (0.3-0.4 ml for P8 mice; $0.7-0.8 \mathrm{ml}$ for P21 mice) until inflation volumes of $0.3-0.4$ and $0.7-0.8 \mathrm{ml}$ were achieved at P8 and P21, respectively. After the agarose was cooled and allowed to gel, the entire lung was removed, placed on a vibratome, sliced into $300-\mu \mathrm{m}$ sections, and immersed in DMEM solution for overnight incubation $\left(5 \% \mathrm{CO}_{2}\right.$; $37^{\circ} \mathrm{C}$ ). The following day the lung slices were rinsed in Hanks' balanced salt solution (HBSS) and mounted in an in vitro recording chamber for live imaging of airway responses to methacholine challenge. Slices were obtained from the left lobe of each animal and sectioned transversely from lateral to medial.

For live imaging of individual airways, the slices were held covered with a thin lightweight sheet of mesh, which was also held in place with petroleum jelly. The recording chamber containing the slice was mounted on a microscope (Leica DMLFS) and perfused (7 ml/min) continuously with HBSS using a perfusion pump (MPII Harvard Instruments). The microscope mounted with a camera (Rolera Fast, Q Imaging, Canada) was used to identify individual airways under high magnification $(\times 5)$. After an initial 3-min baseline period, the lung slice and chamber were perfused with increasing concentrations of methacholine and continuous infusion of HBSS at $7 \mathrm{ml} / \mathrm{min}$ was maintained both prior to and during methacholine application. The extent of constriction in response to methacholine doses of $0.25,0.5,1,2,4$, and $8 \mu \mathrm{m}$ was determined from computer software (Image) imaging of the luminal surface area at the end of a 2-min drug exposure. The agarose that had been perfused into the lungs to allow for cutting of the slice is often dislodged from the airway during the cutting procedure of overnight incubation. The presence of agarose in the airway was examined under a microscope before the methacholine challenge was performed, and those airways containing agarose were excluded from the study as this may have influenced airway caliber.

\section{Airway Immunohistochemistry and Alveolar Morphometry}

Immunohistochemistry for $\alpha$-smooth muscle actin was performed as previously described [11], employing a monoclonal antibody against mouse anti- $\alpha$-smooth muscle actin (1:400 dilution; Sigma-Aldrich). Immune complexes were captured with FITCconjugated donkey anti-mouse secondary antibodies (1:500; Alexa 


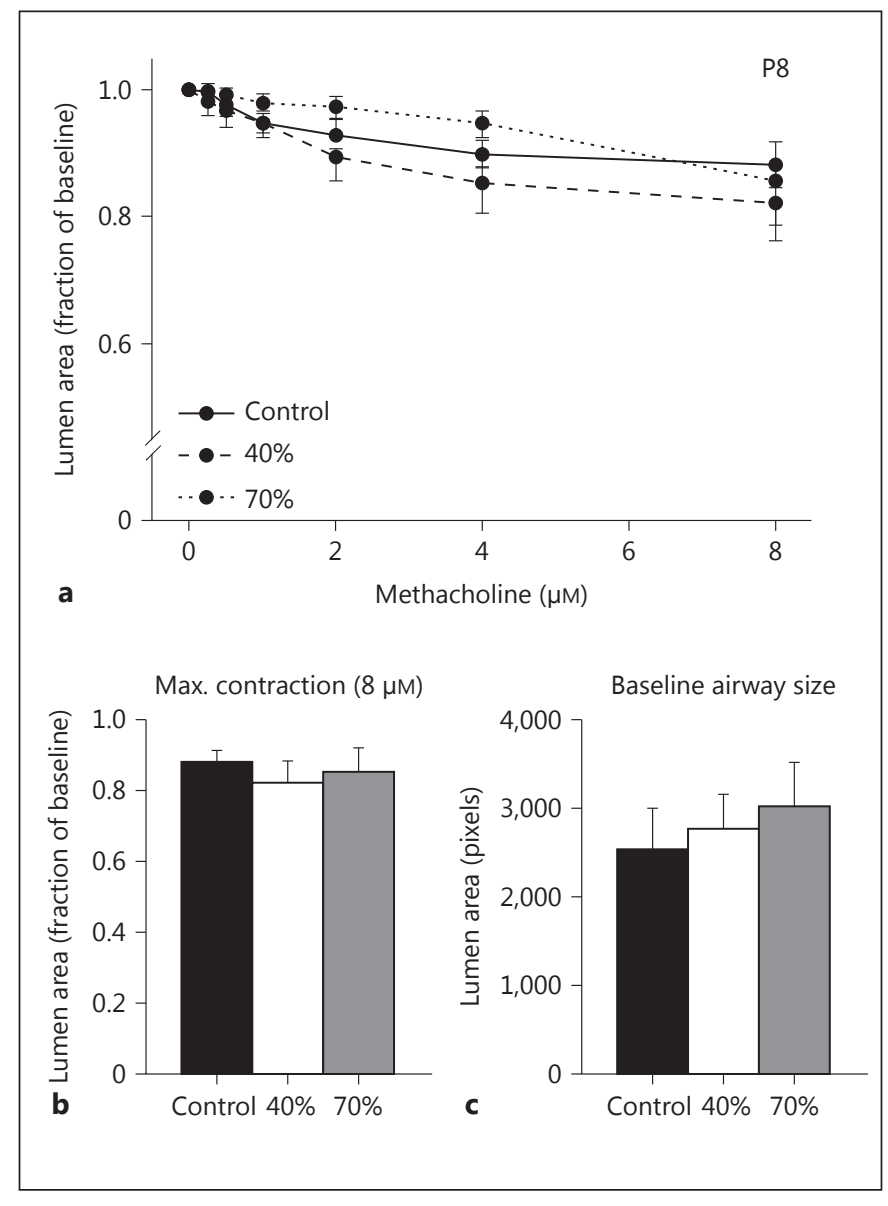

Fig. 1. Airway responses to methacholine challenge in the in vitro living lung slice preparation from $\mathrm{P} 8$ mice $(\mathbf{a}, \mathbf{b})$ that received 1 week of neonatal (P1-P7) hyperoxia ( 40 or $70 \%$ oxygen). Measurements were made on similar size airways in each of the 5 pups for each group (c). Airway responses to methacholine challenge were similar between treatment groups. Means \pm SEM; $1 \mu \mathrm{m}=0.39$ pixels.

Fluor-488; Invitrogen). Individual airways were imaged (Rolera XR CCD camera Q Imaging) and the amount of smooth muscle actin area $\left(\mu \mathrm{m}^{2}\right)$ was analyzed with software (Image-Pro Plus 7.0; Media Cybernetics, Silver Spring, Md., USA). The green fluorescent areas of $\alpha$-smooth muscle actin area were normalized for airway size using the basement membrane circumference.

Myosin light chain (MLC) immunohistochemistry employed rabbit anti-MLC polyclonal antibody specific for phosphorylated MLC (1:500; ab2480 Abcam, Cambridge, Mass., USA). The secondary antibody was biotinylated goat anti-rabbit (1:500; Jackson ImmunoResearch, West Grove, Pa., USA) followed by an ABC reagent, visualized by diaminobenzidine (Vectastain; Vector Laboratories, Burlingame, Calif., USA). Primary antibody was omitted for negative control. The MLC area was also normalized to the basement membrane perimeter.

Airway Hyperreactivity Is Delayed after Mild Neonatal Hyperoxic Exposure

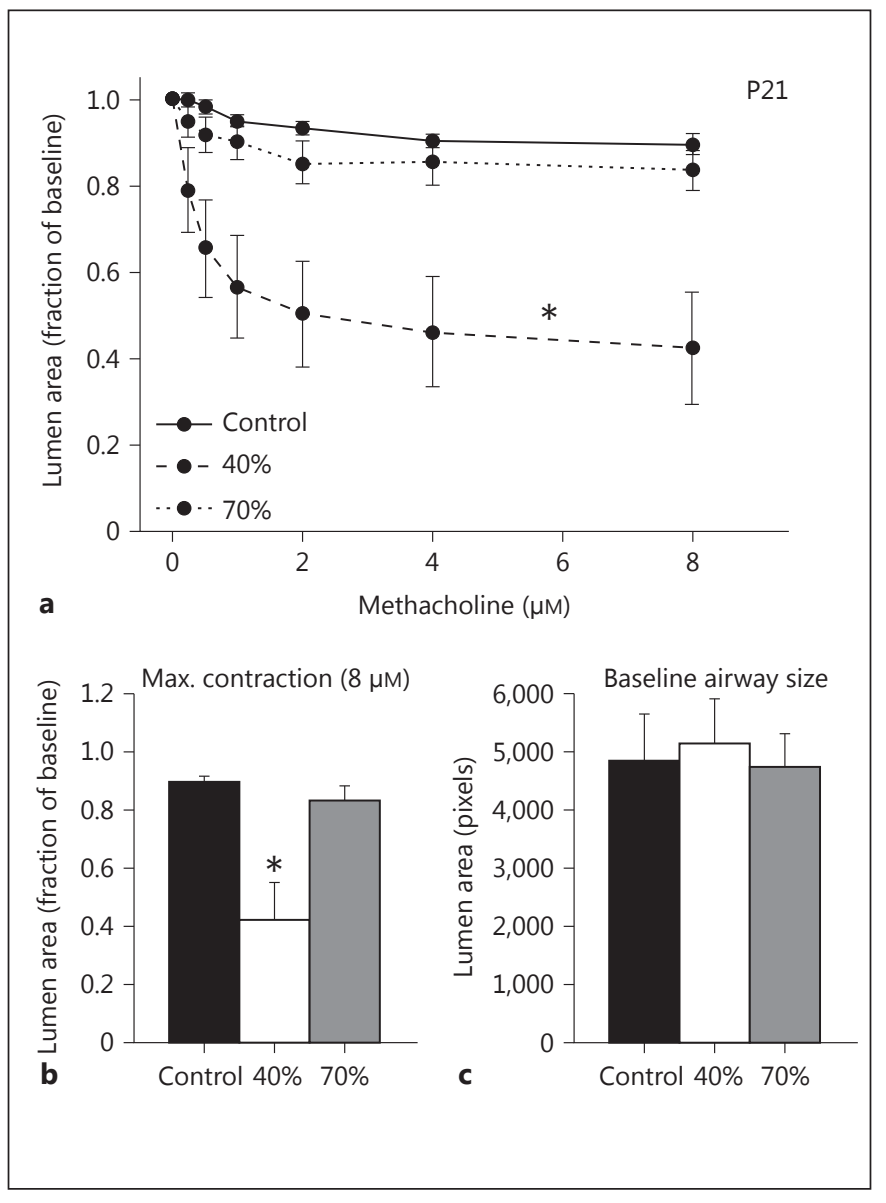

Fig. 2. Airway responses to methacholine challenge in the in vitro living lung slice preparation in P21 mice $(\mathbf{a}, \mathbf{b})$ that received 1 week of neonatal (P1-P7) hyperoxia (40 or $70 \%$ oxygen). Measurements were made on similar size airways in each of the five pups for each treatment group (c). Note: there was an augmented airway constriction in $40 \%$ oxygen-exposed mice compared to control, whereas $70 \%$ exposure had no effect on airway reactivity. ${ }^{*}$ Significant difference from control mice $(\mathrm{p}<0.05)$. Means \pm SEM; $1 \mu \mathrm{m}=0.39$ pixels.

Hematoxylin-eosin-stained sections were used to assess radial alveolar counts as we have previously described [11] by counting the number of alveolar septa transected by a perpendicular line drawn from the terminal bronchioles to the nearest connective tissue septum.

\section{Statistics}

Statistical comparison of airway reactivity to methacholine and body weight was performed between treatment groups using a twoway, repeated-measure ANOVA for each age group or between age groups where appropriate. Comparison of airway and lung morphology (area of actin and MLC, radial alveolar counts) in each age group was performed using a one-way ANOVA with a StudentNewman-Keuls post hoc test. Differences were considered significant at $\mathrm{p}<0.05$. All values are expressed as means \pm 1 SEM. 
Fig. 3. Comparison of airway responses to methacholine $(8 \mu \mathrm{M})$ between P8 and P21 mice for each treatment group. Note: the magnitude of the response to methacholine (no differences between slopes, left panel) was similar between P8 and P21 control mice, whereas airway reactivity was significantly higher at P21 (steeper slope, middle panel) compared to P8 following 40\% oxygen exposure. $70 \%$ oxygen exposure had similar effects on P8 and P21 airway reactivity (right panel). " Significant difference in the slope compared to P8 mice $(\mathrm{p}<0.05)$. Means \pm SEM; $1 \mu \mathrm{m}=0.39$ pixels.

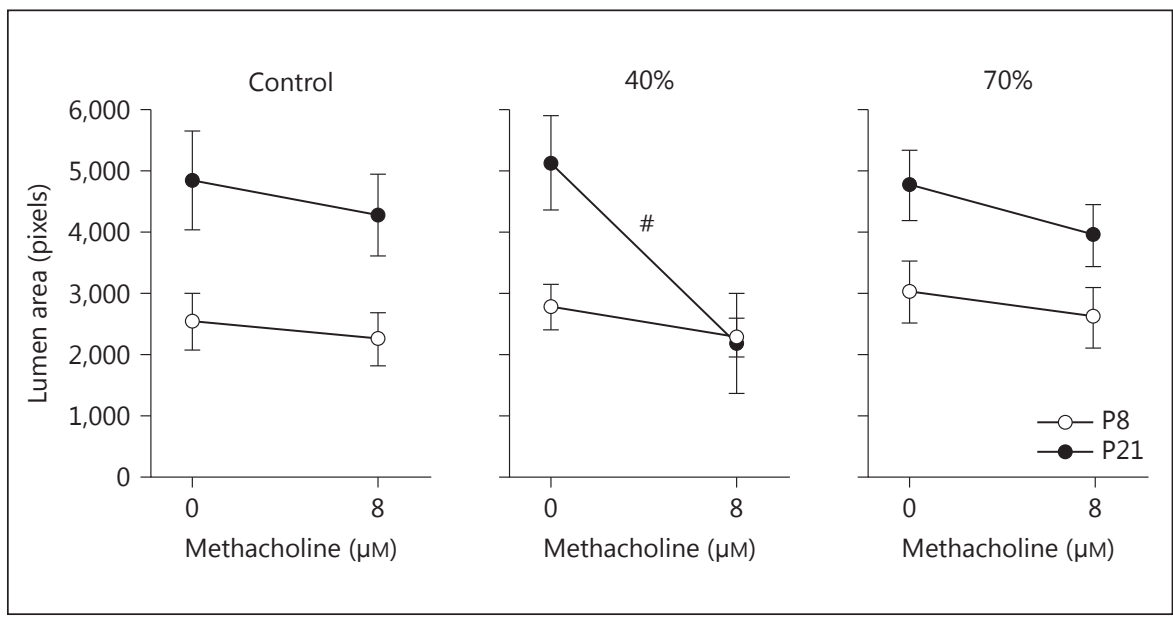

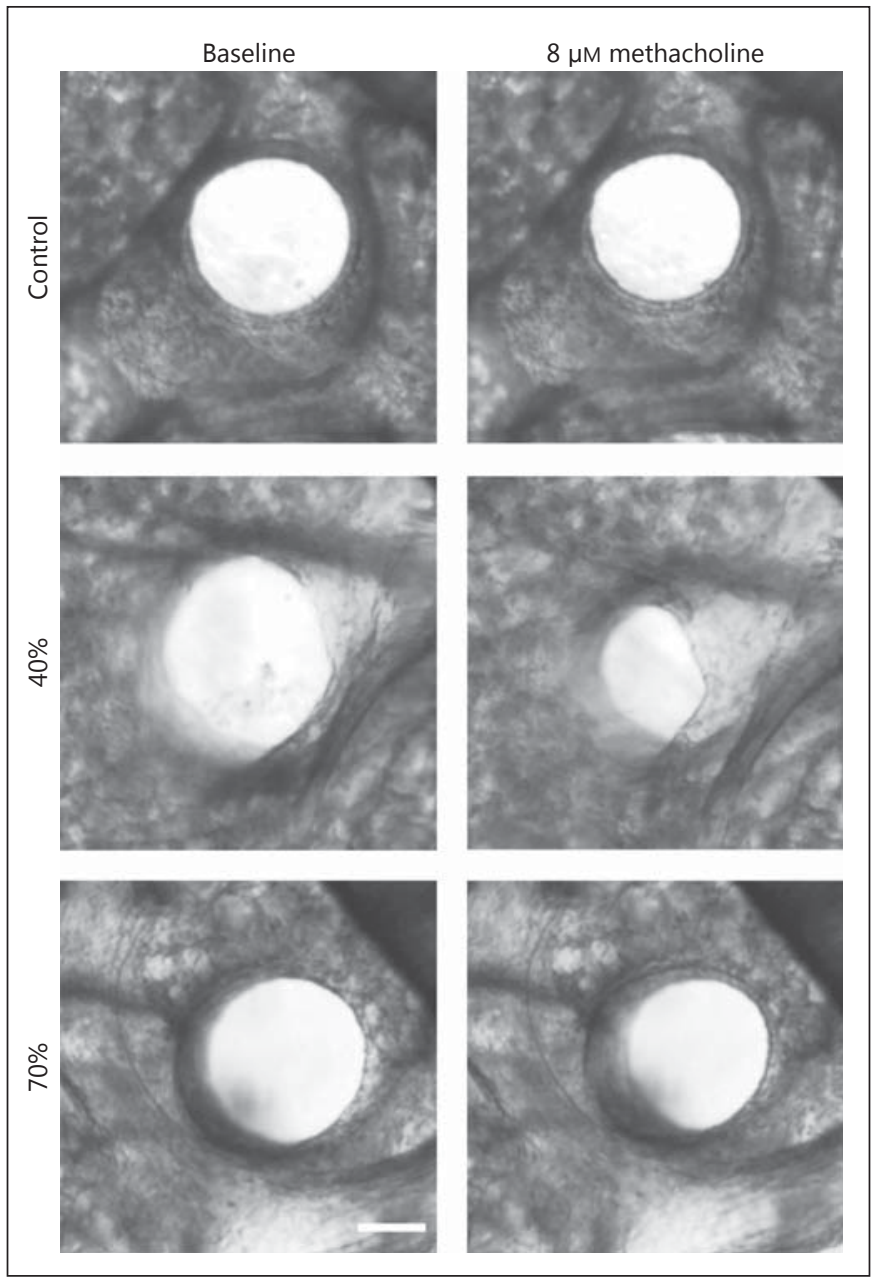

Fig. 4. Representative contractile responses in airways from P21 mice previously exposed to room air (control) or 40 or $70 \%$ oxygen. Note: the greater decrease in lumen size in the $40 \%$ exposed tissue in response to $8 \mu \mathrm{M}$ methacholine. Scale bar $=100 \mu \mathrm{m}$.

\section{Results}

Airway Reactivity to Methacholine Challenge in 1and 3-Week-Old Mice

Airway responses to methacholine challenge in P8 male mice are provided in figure 1 . The slope of the airway response and maximal contraction to increasing doses of methacholine were not significantly different between treatment groups (fig. 1a, b). For consistency, airways of similar size were chosen as indicated by the comparable baseline airway lumen area (fig. 1c). These data suggest that a week of mild ( $40 \%$ oxygen) or severe (70\% oxygen) neonatal hyperoxia exposure does not affect airway responses to methacholine challenge. In contrast, mild hyperoxia increased airway reactivity to methacholine at P21, 2 weeks after treatment ended, as indicated by the larger decrease in airway luminal area (fig. 2a, b) compared to control mice. However, airway responses to methacholine were similar between control mice and severe hyperoxia exposure.

Control mice raised in room air were equally reactive to methacholine $(8 \mu \mathrm{M})$ at P8 compared to 3 -week-old mice (fig. 3, left panel). However, 3-week-old mice exposed to mild hyperoxia exhibited an enhanced airway response at P21 than at 1 week, as indicated by the steeper slope of the response to maximum methacholine challenge (fig. 3, middle panel). In contrast, the slope of the response to methacholine was similar between 1and 3-week-old mice exposed to severe hyperoxia. Representative contractile responses at P21 are shown in figure 4 after room air (control) or 40 or $70 \%$ oxygen exposure. These data indicate that mild neonatal hyperoxia does not affect airway reactivity at the time of ex- 
Fig. 5. Immunohistochemical quantification of $\alpha$-smooth muscle ( $\alpha \mathrm{SM}$ ) actin in P8 and P21 mice following neonatal hyperoxia exposure. The aSM actin area expressed per airway (AW) basement membrane (BM) was similar between treatment groups at P8 (a), whereas at P21, it was increased by $40 \%$ but not $70 \%$ oxygen (c). Representative images are shown in $\mathbf{b}$ and d. * Significant difference from control and $70 \%$ exposed mice $(\mathrm{p}<0.05)$. Means \pm SEM. Ctrl = Control.

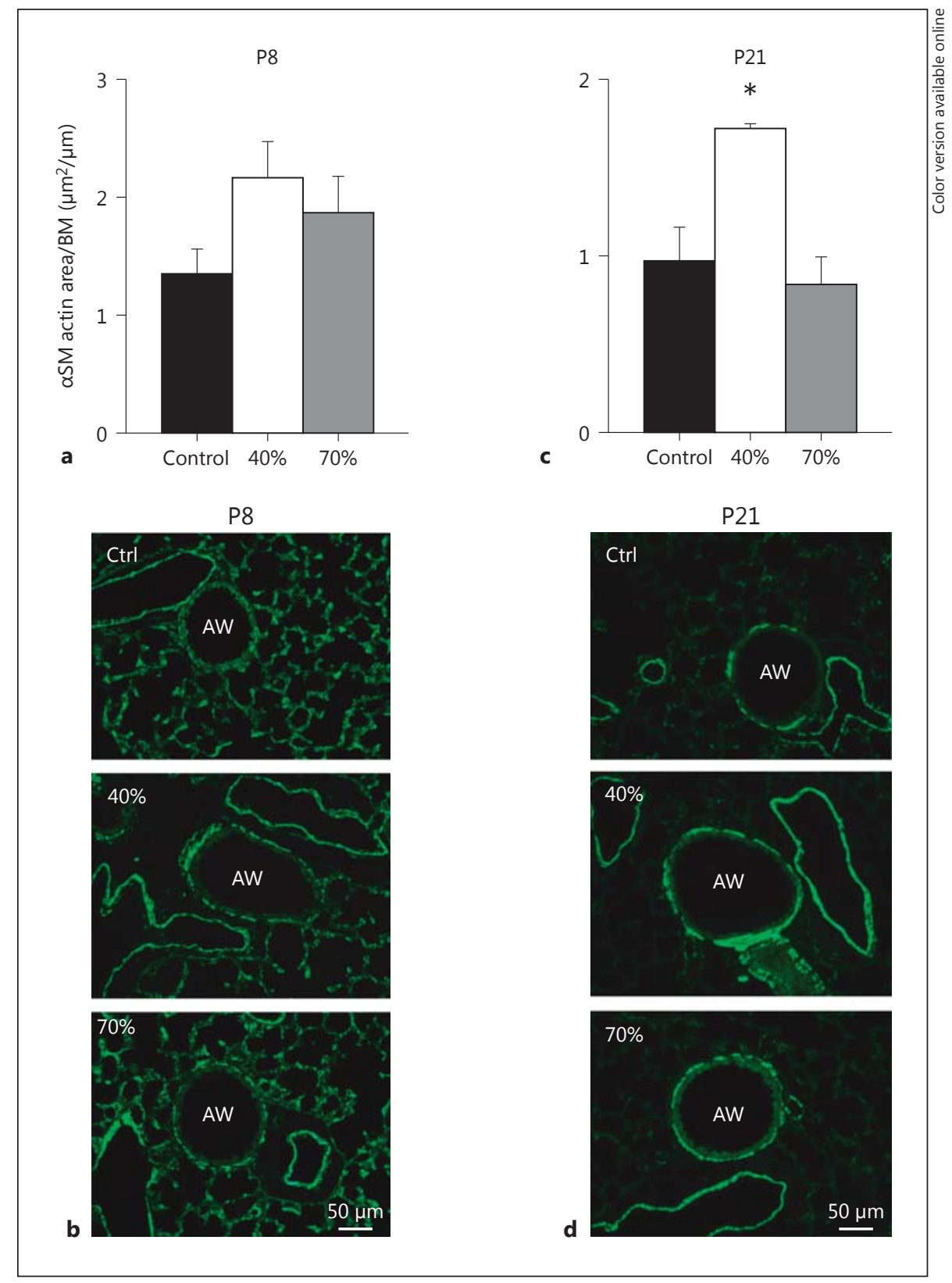

posure, but rather elicits a delayed effect that manifests approximately 2 weeks after treatment.

\section{$\alpha$-Smooth Muscle Actin, MLC, and Alveolar \\ Morphology}

At P8, there was no significant effect of oxygen exposure on $\alpha$-smooth muscle actin expression (fig. 5a). At P21, oxygen exposure did have an overall effect on actin expression with a significant increase after $40 \%$ exposure when compared to both the room air control and $70 \%$ oxygen groups (fig. 5c). Consistent with actin expression, MLC expression at $\mathrm{P} 8$ also did not differ between oxygenexposed groups (fig. 6a). At P21, there was also no significant overall effect of treatment on MLC levels, although there was a trend for increased MLC expression after 40 versus $70 \%$ oxygen exposure (fig. 6b). Compared to room air controls, there was a significant and comparable decrease in radial alveolar counts after both 40 and $70 \%$ oxygen exposure which did not differ between P8 and P21 pups (fig. 6c, d). 


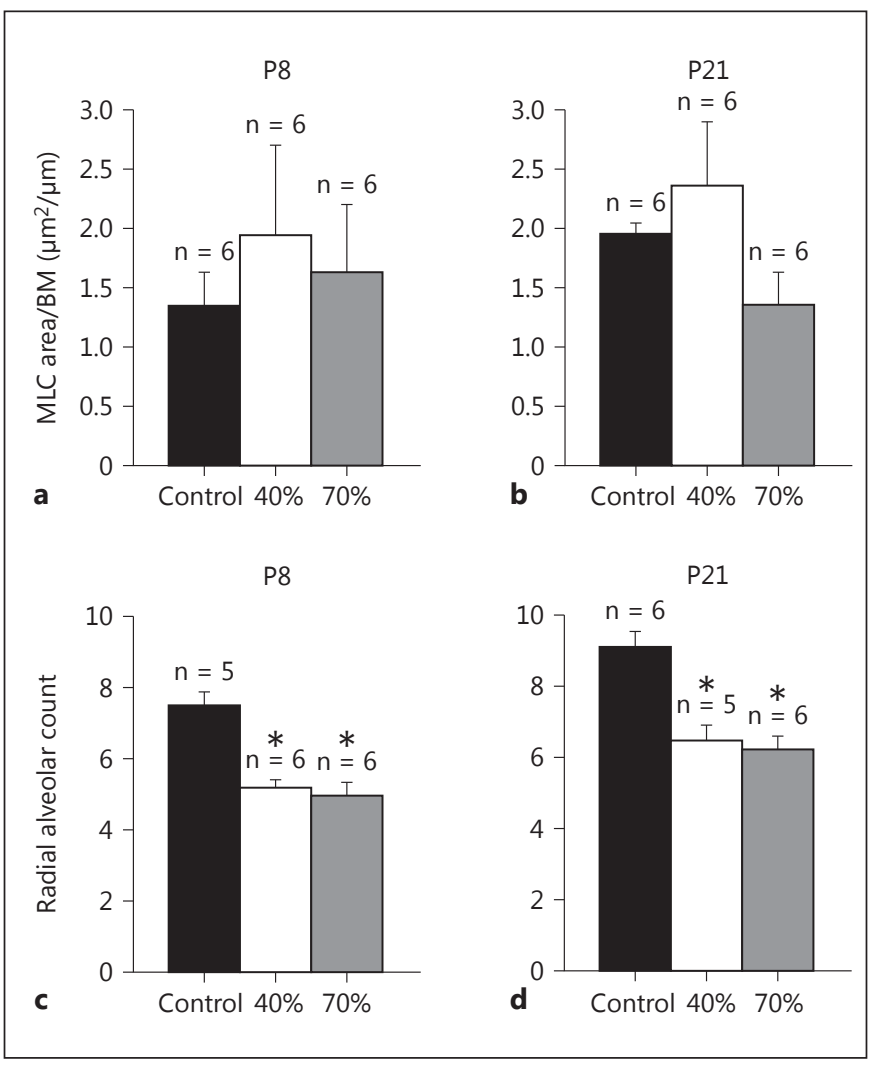

Fig. 6. Immunohistochemical quantification of airway MLC and radial alveolar counts from P8 and P21 mice following neonatal hyperoxia exposure. The amount of MLC, normalized to basement membrane (BM) perimeter length, was similar between treatment groups at P8 and P21 (a, b), but there was a tendency for increased MLC following $40 \%$ oxygen exposure compared to $70 \%$ oxygen in P21 mice. Radial alveolar counts $(\mathbf{c}, \mathbf{d})$ were also reduced at both age groups exposed to either 40 or $70 \%$ oxygen compared to control mice $(*$ both $\mathrm{p}<0.05)$. Means \pm SEM. Ctrl $=$ Control.

\section{Discussion}

Although increased airway reactivity and resultant wheezing disorders are widely recognized clinical problems in former preterm infants, there is limited information on their natural history in the postneonatal period. This is attributable, in part, to the challenges of quantifying in vivo airway reactivity in both human infants and animal models during early postnatal development. Our study was motivated by the widespread clinical observation that airway hyperreactivity typically manifests beyond the neonatal period, especially in infants who do not have a history of BPD. We, therefore, chose to study the time course of airway hyperreactivity in a mouse pup model in response to mild versus severe hyperoxic expo- sure. We hypothesized that the onset of airway hyperreactivity would be delayed after neonatal hyperoxic exposure, and that this might correlate with expression of airway smooth muscle contractile proteins.

We utilized living lung slice preparation which enables in vitro measurement of airway contractions in the presence of bronchoalveolar attachments and cell-matrix interactions $[15,17]$. This technique provides a useful complement to in vivo measurements of airway resistance, especially in small animal models, such as neonatal mice, in which in vivo data of respiratory function with bronchoconstrictors have not been reported prior to 14 postnatal days $[10,11]$. Unfortunately, the widely used flexiVent system for measuring respiratory function requires pups to be at least 7-8 g, making studies prior to approximately 14 days unreliable. It is very reassuring that our slice data at 3 weeks are comparable to our prior lung function studies [11].

Our major finding is that while neither mild nor severe hyperoxia increased airway contraction at P8, airway contractility significantly increased at P21, and only in mice exposed to mild hyperoxia. This occurred 2 weeks after cessation of exposure to $40 \%$ oxygen, suggesting a delayed effect of early neonatal hyperoxia on airway hyperreactivity. We have previously reported in 21-day-old mice that mild, but not severe, hyperoxia increased the response of respiratory system resistance to methacholine [11]. The current study employing lung slices confirms this finding and suggests that the living lung slice technique approximates the in vivo condition. We do recognize that an important limitation of the lung slice technique is the absence of an intact autonomic nervous system and accompanying circulation.

Mild hyperoxic exposure was associated with an increase in airway $\alpha$-smooth muscle actin at P21. We also observed a trend toward increased MLC expression after 40 versus $70 \%$ oxygen exposure at $\mathrm{P} 21$. These findings are consistent with the observation of Hartman et al. [18] in human fetal airway smooth muscle cells that modest hyperoxia has a proliferative effect while severe hyperoxia causes apoptosis. We speculate that apoptosis induced by more severe hyperoxia [19] is the mechanism underlying the greater airway constrictor effect of 40 versus $70 \%$ oxygen exposure at $\mathrm{P} 21$. All preterm infants are at increased risk of wheezing disorders; however, this problem is enhanced in those with a history of BPD $[1,2]$. While such infants may be exposed to higher or longer supplemental oxygen, this is typically accompanied by some form of positive pressure ventilation which was absent from our model of hyperoxic exposures. 
We performed additional histologic studies to begin to identify a mechanism whereby neonatal hyperoxic exposure did not have an immediate effect on airway hyperreactivity. Increased $\alpha$-smooth muscle actin, as a measure of airway smooth muscle mass, only reached significance at P21 after prior $40 \%$ oxygen exposure. We also measured MLC content as a more specific contractile protein and observed no effect of oxygen at P8 and only a trend toward higher expression at $\mathrm{P} 21$ in 40 versus $70 \%$ exposure. Actin may be expressed by airway smooth muscle cells such as fibroblasts and myofibroblasts, which may contribute to constriction, and hence the stronger correlation between our physiologic findings and actin rather than myosin [20]. It is possible that the myosin data may have reached significance with larger group sizes. Future studies might focus on more specific contractile elements in airway smooth muscle and the relative roles of hypertrophy versus hyperplasia in our model.

Recent studies of neonatal lung injury have focused on the role of alveolar simplification in later respiratory morbidity of preterm infants with BPD [21], which might diminish tethering of small airway structures embedded in alveolar walls resulting in increased airway contractile responses [22]. However, radial alveolar counts were decreased to a comparable degree after both levels of hyperoxia at both P8 and P21, indicating it is unlikely that altered alveolar morphology is a major contributor to our findings of delayed airway hyperreactivity after mild hyperoxic exposure. We did not quantify epithelial thickness in our slices; however, all our physiologic responses were based on initial lumen size which we attempted to match between airways. Therefore, any change in epithe- lial thickness in response to hyperoxia should not have contributed to our findings. It should be noted that the lung slice technique does not provide a comparable measure to baseline lung resistance as would be obtained from in vivo pulmonary mechanisms. Three-week-old mouse pups were chosen to approximate the maturational age of human infants who exhibit a high incidence of wheezing following preterm birth. Future studies might address later ages as neonatal hyperoxia increases airway reactivity in mature mice [9] in a sex-dependent manner [16]. Although our prior in vivo study in developing mice did not demonstrate clear sex differences in airway reactivity [11], we chose to study only male pups to avoid confounding our current data with the previously described effect of sex on airway hyperresponsiveness in developing mice [16].

In conclusion, we have documented a delay in development of airway hyperreactivity exhibited by mouse pups exposed to mild hyperoxia. Our data suggest this is associated with greater airway smooth muscle mass, although we cannot exclude other physiologic pathways contributing to the balance of airway contractile/relaxant mechanisms. This study provides new insight into airway-related symptomatology that preterm infants do not exhibit until the postneonatal period.

\section{Acknowledgment}

The National Heart, Lung and Blood Institute, Grant R01HL056470 (R.J.M.), and the Department of Pediatrics, Rainbow Babies and Children's Hospital provided funding for this study.

\section{References}

1 Fawke J, Lum S, Kirkby J, Hennessy E, Marlow N, Rowell V, Thomas S, Stocks J: Lung function and respiratory symptoms at 11 years in children born extremely preterm: the EPICure study. Am J Respir Crit Care Med 2010;182:237-245.

-2 Reyburn B, Martin RJ, Prakash YS, MacFarlane PM: Mechanisms of injury to the preterm lung and airway: implications for long-term pulmonary outcome. Neonatology 2012;101: 345-352.

>3 Harju M, Keski-Nisula L, Georgiadis L, Raisanen S, Gissler M, Heinonen S: The burden of childhood asthma and late preterm and early term births. J Pediatr 2014;164:295-299. e1.

Airway Hyperreactivity Is Delayed after

Mild Neonatal Hyperoxic Exposure
4 McEvoy C, Venigalla S, Schilling D, Clay N, Spitale P, Nguyen T: Respiratory function in healthy late preterm infants delivered at 3336 weeks of gestation. J Pediatr 2013;162:464469.

5 Vrijlandt EJ, Kerstjens JM, Duiverman EJ, Bos AF, Reijneveld SA: Moderately preterm children have more respiratory problems during their first 5 years of life than children born full term. Am J Respir Crit Care Med 2013;187:1234-1240

6 Fayon M, Tayara N, Germain C, Choukroun ML, De La Roque ED, Chene G, Breilh D, Marthan R, Demarquez JL: Efficacy and tolerance of high-dose inhaled ipratropium bromide vs. terbutaline in intubated premature human neonates. Neonatology 2007;91:167-173.
7 Belik J, Jankov RP, Pan J, Tanswell AK Chronic $\mathrm{O}_{2}$ exposure enhances vascular and airway smooth muscle contraction in the newborn but not adult rat. J Appl Physiol 2003;94:2303-2312.

8 Choi CW, Kim BI, Mason SN, Potts-Kant EN, Brahmajothi MV, Auten RL: Intra-amniotic LPS amplifies hyperoxia-induced airway hyperreactivity in neonatal rats. Pediatr Res 2013;74:11-18.

-9 O’Reilly M, Harding R, Sozo F: Altered small airways in aged mice following neonatal exposure to hyperoxic gas. Neonatology 2014; 105:39-45. 
10 Velten M, Heyob KM, Rogers LK, Welty SE: Deficits in lung alveolarization and function after systemic maternal inflammation and neonatal hyperoxia exposure. J Appl Physiol 2010;108:1347-1356.

-11 Wang H, Jafri A, Martin RJ, Nnanabu J, Farver C, Prakash YS, MacFarlane PM: Severity of neonatal hyperoxia determines structural and functional changes in developing mouse airway. Am J Physiol Lung Cell Mol Physiol 2014;307:L295-L301.

12 Yee M, Chess PR, McGrath-Morrow SA, Wang Z, Gelein R, Zhou R, Dean DA, Notter RH, O'Reilly MA: Neonatal oxygen adversely affects lung function in adult mice without altering surfactant composition or activity. Am J Physiol Lung Cell Mol Physiol 2009; 297:L641-L649.

13 Burri PH: Structural aspects of postnatal lung development - alveolar formation and growth. Biol Neonate 2006;89:313-322.
4 Amy RW, Bowes D, Burri PH, Haines J, Thurlbeck WM: Postnatal growth of the mouse lung. J Anat 1977;124:131-151.

15 Sanderson MJ: Exploring lung physiology in health and disease with lung slices. Pulm Pharmacol Ther 2011;24:452-465.

16 McKenzie R, Burton MD, Royce SG, Tang ML: Age and sex influences on airway hyperresponsiveness. J Asthma 2010;47:651-654.

17 Donovan C, Royce SG, Esposito J, Tran J, Ibrahim ZA, Tang ML, Bailey S, Bourke JE: Differential effects of allergen challenge on large and small airway reactivity in mice. PLoS One 2013;8:e74101.

18 Hartman WR, Smelter DF, Sathish V, Karass M, Kim S, Aravamudan B, Thompson MA, Amrani Y, Pandya HC, Martin RJ, Prakash YS, Pabelick CM: Oxygen dose responsiveness of human fetal airway smooth muscle cells. Am J Physiol Lung Cell Mol Physiol 2012;303:L711-L719.
9 Yi M, Masood A, Ziino A, Johnson BH, Belcastro R, Li J, Shek S, Kantores C, Jankov RP, Tanswell AK: Inhibition of apoptosis by $60 \%$ oxygen: a novel pathway contributing to lung injury in neonatal rats. Am J Physiol Lung Cell Mol Physiol 2011;300:L319L329.

20 Singh SR, Hall IP: Airway myofibroblasts and their relationship with airway myocytes and fibroblasts. Proc Am Thorac Soc 2008;5:127132.

21 Sosenko IRS, Bancalari E: New developments in the presentation, pathogenesis, epidemiology and prevention of bronchopulmonary dysplasia; in Polin RA, Bancalari E (eds): The Newborn Lung. Philadelphia, Saunders, 2010, pp 187-207.

22 Colin AA, McEvoy C, Castile RG: Respiratory morbidity and lung function in preterm infants of 32 to 36 weeks' gestational age. Pediatrics 2010;126:115-128 